\title{
Inflammatory Reactions Induced by PTFE Tapes (Study in Rabbits)
}

\author{
Fausto Viterbo ${ }^{1, *}$, Túlio Magno de Melo Caldonazo ${ }^{2}$ and Maria Aparecida Custódio Domingues ${ }^{3}$ \\ ${ }^{1}$ Plastic Surgery Full Professor, Department of Surgery, Sao Paulo State University, Botucatu, Brazil \\ ${ }^{2}$ Academic, Sao Paulo State University, Botucatu, Brazil \\ ${ }^{3}$ Pathology Assistant Professor, Department of Pathology, Sao Paulo State University, Botucatu, Brazil
}

*Corresponding author: Viterbo F, Plastic Surgery Full Professor, Department of Surgery, Sao Paulo State University, Botucatu, Brazil, Tel: +55 1499671 2315; E-mail: faustoviterbo@hotmail.com

Received: 21 Nov, 2019 | Accepted: 09 Dec, 2019 | Published: 13 Dec, 2019

Citation: Viterbo F, Caldonazo TMM, Domingues MAC (2019) Inflammatory Reactions Induced by PTFE Tapes (Study in Rabbits). J Surg Open Access 6(1): dx.doi.org/10.16966/2470-0991.203

Copyright: (C) 2019 Viterbo F, et al. This is an open-access article distributed under the terms of the Creative Commons Attribution License, which permits unrestricted use, distribution, and reproduction in any medium, provided the original author and source are credited.

\author{
Abstract \\ Purpose: To evaluate the inflammatory response due to the use of ultra-thin PTFE tapes.
}

Methods: Ten Norfolk rabbits were submitted a surgical procedure. They received $60 \mathrm{~mm}$ (long) $\times 18 \mathrm{~mm}$ (wide) $\times 0.05 \mathrm{~mm}$ (thick) PTFE tape implants in the ears and inguinal region. After 3 months, the material was extracted from the implantation site for histological analysis. Each sample was analyzed with presence or absence of inflammation. It was characterized by type (acute or chronic-whether chronic, granulomatous or not), amount (mild, intense or moderate) and type of leukocyte infiltrate (eosinophils, lymphocytes, neutrophils or macrophages). In addition, neovasculogenesis and fibrosis of the lesion were evaluated for their presence and intensity.

Results: From the statistical analysis of the histology of the slides, we noticed predominantly: characteristic standard of chronic type inflammation, with mild to moderate intensity and presence of granulomatous response (foreign-giant cells).

Conclusion: It can be concluded that the use of ultra-thin PTFE tape as an implant in animals presented: mild to moderate inflammatory response process of chronic granulomatous type, with the presence of mild to moderate neovasculogenesis and with the presence of mild to moderate fibrosis in the region.

Keywords: Plastic surgery; Polytetrafluoroethylene; Facial injuries

\section{Introduction}

Cases of facial deformities are divided into two distinct groups: congenital or traumatic facial deformities. The group of acquired deformities includes infectious processes, trauma and surgical or radiotherapeutic procedures of neoplasms. On the other hand, the group of traumatic causes is highlighted by the significant increase in the incidence of motor vehicle accidents, according to a study involving 5790 motorcyclists injured in accidents, showed that $24.4 \%$ had facial trauma [1].

Most of these lesions generate deformities in skin and skeletal tissues. To treat these situations, autologous tissue grafts (such as adipose and bone) or alloplastic materials (such as silicone and acrylic) are used. The positive aspect of the fat tissue grafts is that they are autologous and do not trigger an inflammatory reaction, but require local anesthesia in the donor and recipient areas, and have unpredictable absorption; and, in most cases, need other subsequent procedures.

Alloplastic materials (for example, silicone, Polytetrafluoroethylene (PTFE) and acrylic), on the other hand, have very significant positive points as: not having to address the donor area, decreased procedure time as it does not require graft removal, unlimited amount and the possibility of being adapted in shape and volume for each patient. One of the most commonly used materials as alloplastic is medical silicone, which is used in slides, solid blocks or in the form of gel coated with a silicone slide, which is widely used in breast and gluteal augmentation procedures [2]. Another alloplastic material, PTFE, is becoming more significant in cardiac valve replacement, abdominal wall reconstruction, and facial implant replacement procedures [3]. According to some studies, PTFE has a good inflammatory response compared to silicone [4].

Thus, one of the main focuses of research on biomaterials is to broaden knowledge and understanding about cellular responses and inflammatory processes from implanted materials, in order to optimize implant's quality and function. Several thermoplastic polymers, such as polytetrafluoroethylene (PTFE), have hydrophobic character, inducing less inflammatory response and, therefore, are less harmful to patients [5].

For minor deformities with age-accentuated sags and grooves, the current treatment is fat injections or injections of alloplastic substances such as PMMA (Polymethyl Methacrylate) or hyaluronic acid. It has the disadvantage of provoking inflammatory and allergic reactions in some patients, as well as causing long-term deforming 
inflammatory reactions and fibroses in many patients, and its use for this purpose has been almost banned. Both have another important disadvantage that is the impossibility of being removed. PTFE has been used in solid, soft form and soft blades, but not in ultra-thin tapes. Thinking about the advantages of PTFE, we decided to adopt it in the form of ultra thin-tapes, which would present low tissue reaction and could be removed.

\section{Objective}

To evaluate the inflammatory response due to the use of ultra-thin PTFE tapes.

\section{Methods}

The experimental study was performed at the Sao Paulo State Experimental Research Unit (UNIPEX-UNESP), after approval by Botucatu Medical School Animal Experimentation Ethics Committee (No. 1250/2017-CEUA).

Ten Norfolk rabbits were used, approximately with 70 days old, all male (in order to avoid hormonal changes, which is more common in females). The animals had an approximate weight of $2000 \mathrm{gr}$ and were supplied by the Sao Paulo State University Central BioteriumBotucatu, Sao Paulo.

\section{Surgical procedure}

The animals were submitted to the surgical procedure after anesthesia with Ketamine (Dopalen $\left.{ }^{\circ}\right) 35 \mathrm{mg} / \mathrm{kg}$ and Xylazine (Anasedan $\left.{ }^{\circ}\right) 5 \mathrm{mg} /$ $\mathrm{kg}$ intramuscularly, with local anesthesia using Bupivacaine $7 \mathrm{mg} / \mathrm{kg}$ (solution containing $15 \mathrm{ml}$ serum and $5 \mathrm{ml}$ Bupivacaine $-5 \mathrm{mg} / \mathrm{ml}$ ). When further analgesia was required, $1 / 3$ of the initial intravenous Ketamine dose was administered. Intraoperatively, morphine was administered intramuscularly $(5 \mathrm{mg} / \mathrm{kg}$ ), which provided intense analgesia to animals to control postoperative pain. In the immediate postoperative period, Meloxican $1.5 \mathrm{mg} / \mathrm{kg}$ was used subcutaneously every 24 hours for 3 days.

The animals received $60 \mathrm{~mm}$ (long) $\times 18 \mathrm{~mm}$ (wide) $\times 0.05 \mathrm{~mm}$ (thick) PTFE tape implants in the ears and inguinal region.

Trichotomy and antisepsis were performed with degerming (2\%) and alcoholic (0.5\%) chlorhexidine in ear and inguinal region.

A $1 \mathrm{~cm}$ incision was made in the skin of the inner right ear and the skin tissue was detached from the cartilage to obtain a $2 \mathrm{~cm} \times 1$ $\mathrm{cm}$ spot. In this place of the animal was implanted the PTFE tape. This done, the incision was sutured with single stitches of 5.0 nylon monofilament suture. In all animals, $1 \mathrm{~cm}$ incisions were also made in the right inguinal region and the subcutaneous tissue was dissected close to the skin to obtain a $2 \mathrm{~cm} \times 1 \mathrm{~cm}$ spot. PTFE tape was inserted into this cavity. The incision was sutured in the same way as previously described.

After the procedure, the animals were kept in individual cages appropriate for rabbits, with water and feed suitable (ad libitum), besides preserved circadian cycles. There was no air exhaustion.

All material used during the procedure was sterile to avoid contamination and the animals did not received antibiotics.

The animals were observed weekly for three months for edema formation, presence of pain, hyperemia, abscess formation and partial or total adherence of the implant to deep and superficial planes.

\section{Euthanasia}

After three months the animals were sacrificed at high and increasing doses of Thiopental $90 \mathrm{mg} / \mathrm{kg}$ or Ketamine $105 \mathrm{mg} / \mathrm{kg}$ associated with Xylazine $15 \mathrm{mg} / \mathrm{kg}$ intravenously.

\section{Material extraction}

Material was extracted from the implantation site (ear and inguinal region) for histopathological analysis.

\section{Histopathological analysis}

For histopathological analysis, wedge biopsy was performed of the areas whose ribbons were implanted, with dimensions $2.3 \times 1.3 \times 0.5 \mathrm{~cm}$. The material was fixed in $10 \%$ buffered formalin for further lamination and analysis, in which the presence or absence of inflammation was evaluated. It was characterized by type (acute or chronic whether chronic, granulomatous or not), amount (mild, intense or moderate) and type of leukocyte infiltrate (eosinophils, lymphocytes, neutrophils or macrophages). In addition, neovasculogenesis and fibrosis of the lesion were evaluated for their presence and intensity.

\section{Statistical analysis}

Data from the descriptive statistical analysis about frequency distribution were presented as column charts.

\section{Results}

From the statistical analysis of the histology of the slides, we noticed predominantly: characteristic standard of chronic type inflammation, with mild to moderate intensity and presence of granulomatous response (according to the foreign-giant cells shown in figure 1).

The presence of foreign-body giant cells was noted in all cases, and in some cases it was possible to identify other types of leukocyte infiltrate (as graph 1 shows), such as eosinophilic (Figure 2) and lymphocyte. It is also possible to identify neovasculogenesis process with predominance of mild to moderate degree (as shown in figure 3; and graph 2). It was also noted the predominance of mild to moderate degree of fibrosis in the analyzed slides (as shown in graph 3 ). Finally, the appearance of PTFE tapes at the site of implantation can be clearly seen (as shown in figure 4).

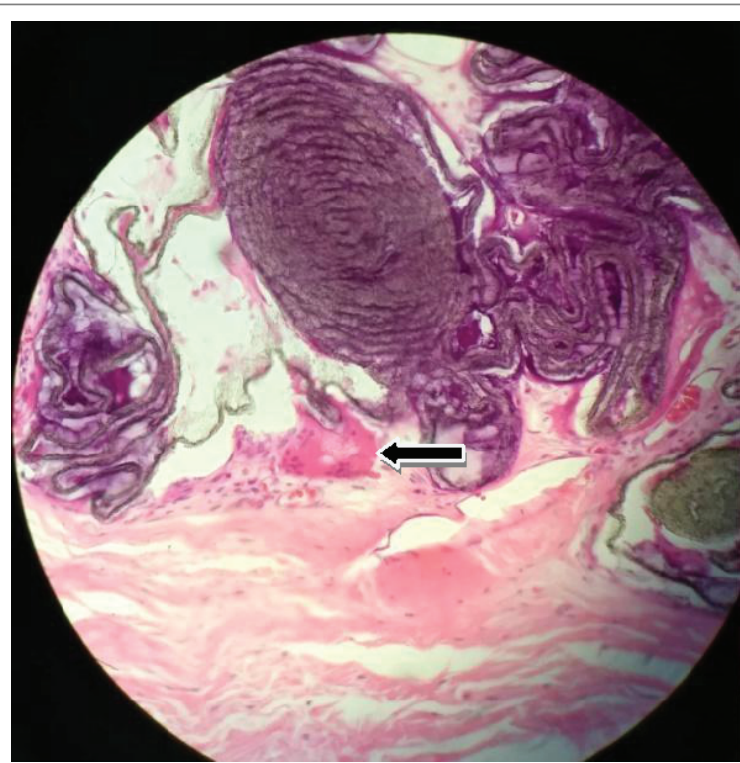

Figure 1: Presence of foreign-body giant cell in implant region. 


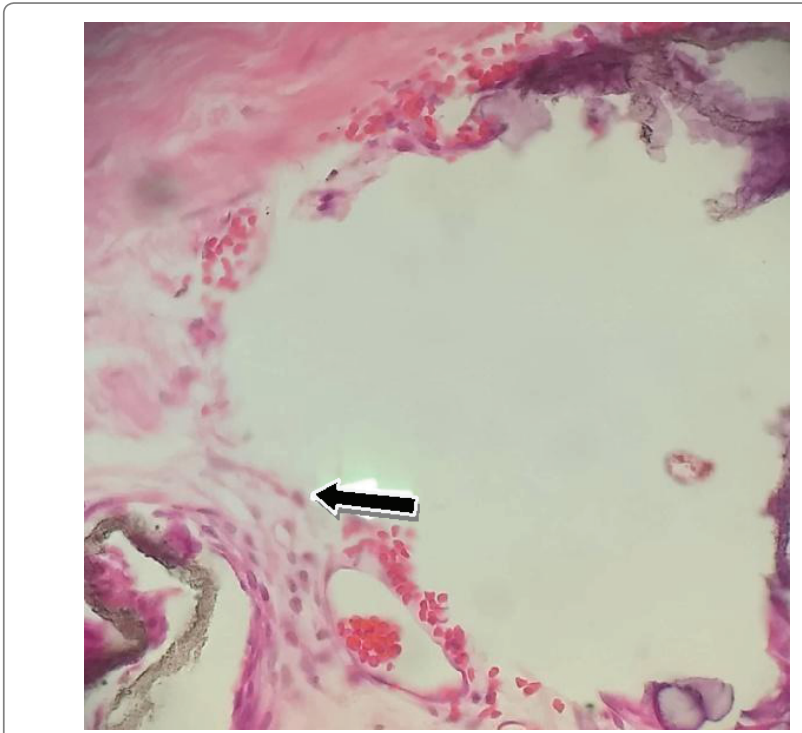

Figure 2: Presence of eosinophils near the implant region.

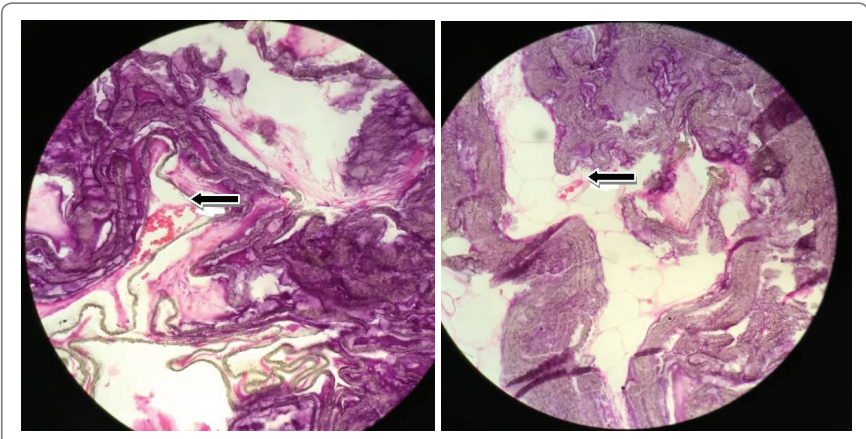

Figure 3: Neovasculogenesis in the implant region.
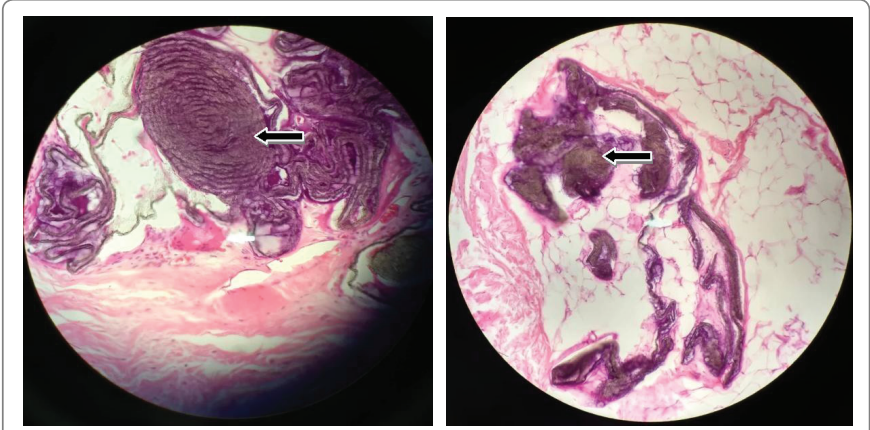

Figure 4: Aspect of PTFE implant in inguinal region.

\section{Discussion}

Experimental studies with alloplastic materials are increasingly pointing to the therapeutic potential of these materials as a fundamental tool for facial deformity repair.

The ability to be removable is a major component that should be analyzed in the surgeon's decision, as studies show cases of complications arising from them [6].

Studies with PTFE have shown that it is a material with a favorable inflammatory response and has an adequate safety profile for use as a facial implant $[7,8]$.

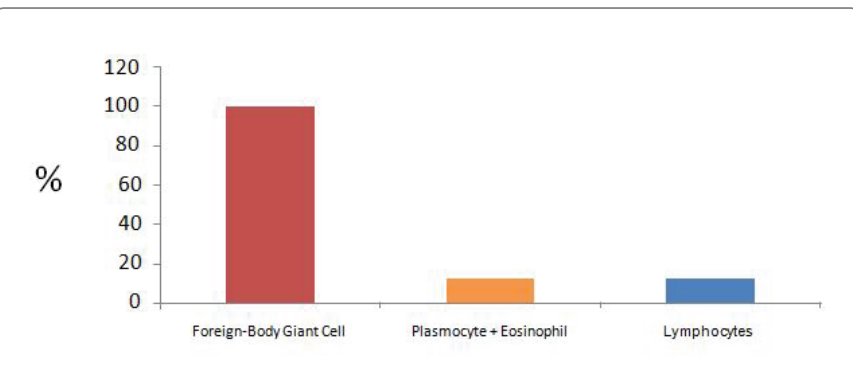

Graph 1: Leukocyte Infiltrate.

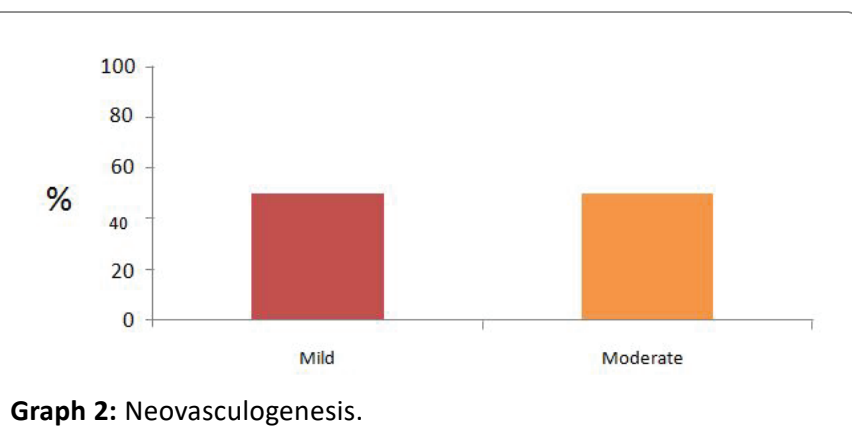

Graph 2: Neovasculogenesis.

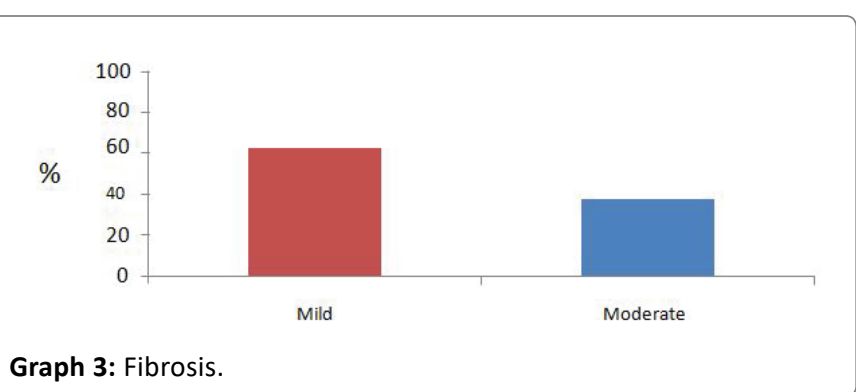

Moreover, in comparative studies with other materials, it is remarkable that PTFE is gaining space in this scenario. Considering that it can generate less inflammatory and less neovasculogenic dynamic when compared to different materials (such as PMMA and silicone) $[9,10]$. Also it is noteworthy the forms of PTFE with new components, such as the addition of Fluorinated Ethylene Propylene (FEPRePTFE) [10,11].

However, the scientific literature lacks experimental and clinical studies with focus in ultra-thin PTFE tapes as a facial implant.

In this context, the ultra-thin tapes have as main attribute the fact that they are superior to other forms of PTFE in the approaches of grooves and small depressions, as they provide better contour and modeling in the place where they will be implanted.

Therefore, it is concluded that ultra-thin PTFE tapes are extremely promising, as they allow not only a reduced inflammatory response, but above all a significant esthetic benefit.

\section{Conclusion}

It can be concluded that the use of ultra-thin PTFE tape as an implant in animals presented: mild to moderate inflammatory response process of chronic granulomatous type, with the presence of mild to moderate neovasculogenesis and with the presence of mild to moderate fibrosis in the region. 


\section{Conflict of Interest}

None.

\section{Financial Source}

São Paulo Research Foundation-FAPESP (2017/25330-4).

\section{References}

1. Bachynski KE (2012) Playing Hockey, Riding Motorcycles, and the Ethics of Protection. Am J Public Health 102: 2214-2220.

2. Herink C, Zwaka PA, Schön MP, Mempel M, Seitz CS (2013) Serious complications following gluteal injection of silicone. Hautarzt 64: 599-602.

3. Panossian A, Garner WL (2004) Polytetrafluoroethylene facial implants: 15 years later. Plast Reconstr Surg 113: 347-349.

4. Ugurbas SC, Kocer NE, Oto S, Kahraman B, Akova YA (2010) Comparison of alloplast materials in experimental extraocular muscle surgery. Ophthalmic Res 44: 50-56.

5. Power KA, Fitzgerald KT, Gallagher WM (2010) Examination of cellhost-biomaterial interactions via high-throughput technologies: A re-appraisal. Biomaterials 31: 6667-6674.
6. Maas CS, Papel ID, Greene D, Stoker DA (1997) Complications of injectable synthetic polymers in facial augmentation. Dermatol Surg 23: 871-877.

7. Shadfar S, Farag A, Jarchow AM, Shockley WW (2015) Safety and Efficacy of Expanded Polytetrafluoroethylene Implants in the Surgical Management of Traumatic Nasal Deformity. JAMA Otolaryngol Head Neck Surg 141: 710-715.

8. Greene D, Pruitt L, Maas CS (1997) Biomechanical effects of e-PTFE implant structure on soft tissue implantation stability: a study in the porcine model. Laryngoscope 107: 957-962.

9. Binder WJ, Dhir K, Joseph J (2013) The role of fillers in facial implant surgery. Facial Plast Surg Clin North Am 21: 201-211.

10. Batniji RK, Hutchison JL, Dahiya R, Lam SL, Williams EF (2002) Tissue response to expanded polytetrafluoroethylene and silicone implants in a rabbit model. Arch Facial Plast Surg 4: 111-113.

11. Sclafani AP, Romo T (2000) Biology and chemistry of facial implants. Facial Plast Surg 16: 3-6. 